\title{
Measurement and simulation of the impact of coherent synchrotron radiation on the Jefferson Laboratory energy recovery linac electron beam
}

\author{
C. C. Hall, ${ }^{*}$ S. G. Biedron, A. L. Edelen, and S. V. Milton \\ Colorado State University, Fort Collins, Colorado 80523, USA \\ S. Benson, D. Douglas, R. Li, and C. D. Tennant \\ Thomas Jefferson National Accelerator Laboratory, Newport News, Virginia 23606, USA \\ B. E. Carlsten \\ Los Alamos National Laboratory, Los Alamos, New Mexico 87545, USA
}

(Received 5 September 2014; published 9 March 2015)

\begin{abstract}
In an experiment conducted on the Jefferson Laboratory IR free-electron laser driver, the effects of coherent synchrotron radiation (CSR) on beam quality were studied. The primary goal of this work was to explore CSR output and effect on the beam with variation of the bunch compression in the IR recirculator. Here we examine the impact of CSR on the average energy loss as a function of bunch compression as well as the impact of CSR on the energy spectrum of the bunch. Simulation of beam dynamics in the machine, including the one-dimensional CSR model, shows very good agreement with the measured effect of CSR on the average energy loss as a function of compression. Finally, a well-defined structure is observed in the energy spectrum with a feature in the spectrum that varies as a function of the compression. This effect is examined in simulations, as well, and a simple explanation for the variation is proposed.
\end{abstract}

DOI: 10.1103/PhysRevSTAB.18.030706

PACS numbers: 29.20.Ej, 41.60.Ap

\section{INTRODUCTION}

The Thomas Jefferson National Accelerator Facility (JLAB) energy recovery linac (ERL) IR free-electron laser (FEL) driver [1] consists of a superconducting radio frequency (SRF) linac, allowing for $\mathrm{cw}$ operation, and a recirculating transport system. This recirculation of the beam back to the linac, after it has passed through the magnetic wiggler, used to generate an IR free-electron laser, allows for recapture of the rf energy before the beam is dumped. The IR FEL can operate at a repetition rate of $75 \mathrm{MHz}$ with a charge per bunch of up to $135 \mathrm{pC}$ providing up to $10 \mathrm{~mA}$ of average current and a beam energy of $160 \mathrm{MeV}$. This gives a very high average power of as much as $10 \mathrm{~kW}$ in the $0.9-10.5 \mu \mathrm{m}$ range, and a high of $14 \mathrm{~kW}$ recorded at $1.6 \mu \mathrm{m}$ from the FEL [2]. ERL operation allows for a much more efficient machine in terms of rf power required; however, this mode of operation necessitates a layout that allows for the electron bunches to recirculate back to the linac (see Fig. 1 for the machine layout) and raises many challenges with respect to maintaining necessary beam quality and suppression of detrimental collective effects that will hamper both FEL efficiency and energy

*chris.hall@colostate.edu

Published by the American Physical Society under the terms of the Creative Commons Attribution 3.0 License. Further distribution of this work must maintain attribution to the author(s) and the published article's title, journal citation, and DOI. recovery. To reduce collective effects in the linac but to maintain high FEL efficiency, it is desirable to start with a low electron bunch current that is then compressed to high peak current just before the FEL. Often, multiple bunch compression stages are used to suppress the impact of coherent synchrotron radiation (CSR) on beam quality. For an ERL-driven FEL, however, the recirculation arcs constrain the placement of a compression section, as compressing before traversing the first arc would degrade beam quality due to CSR emission from the shortened bunch. Because of the high repetition rate and high average current present during operation of the JLAB FEL, the average power of CSR radiation may be as much as $200 \mathrm{~W} / \mathrm{mA}$. This power can produce heating and necessitate that the machine operation be paused periodically to allow for cooling and prevent vacuum pressure rise [2]. Thus the measurement and modeling of CSR in high repetition rate ERL FELs is quite important.

In this paper, we show novel measurements taken on the JLAB FEL. Because of the unique characteristics of this machine, the net bunch compression and point of maximum compression in the machine can be varied through the use of several quadrupoles rather than by the usual method of adjusting the linac phase. The machine can thus be configured to achieve compression starting from acceleration on either the falling or rising side of the rf wave. Measurements were taken over a wide range of compression conditions. Both the impact on the mean energy and the impact on the energy spectrum due to CSR were measured, and comparison to simulations is shown. 


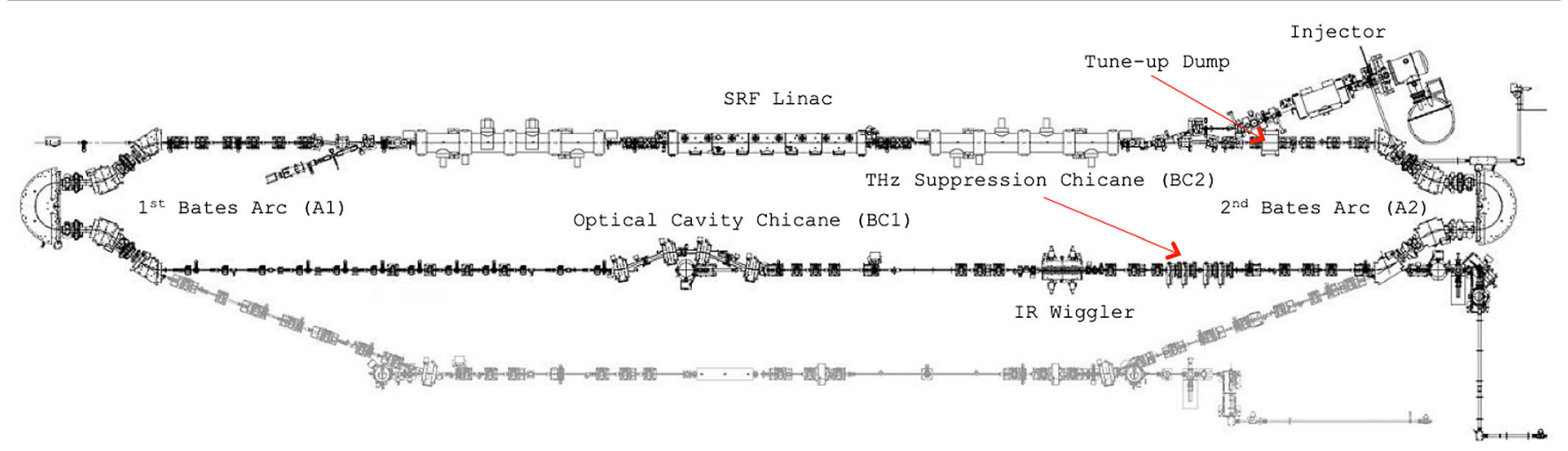

FIG. 1. Layout of the FEL at Jefferson Laboratory. The IR FEL system, shown in black, was used for these measurements. The gray is the UV line and was not used in this experiment.

With this unique setup we are able to show comparisons of measurements of CSR-induced energy loss with simulations in ELEGANT employing the one-dimensional (1D) model of CSR [3]. This comparison shows good agreement with the 1D model over the wide range of compression conditions experienced by the beam and for compression in both the first recirculation arc and the compression chicane.

The energy spectrum displays fragmentation features that, while certainly the result of CSR, appear to not be indicative of the microbunching instability [4,5]. It is seen from simulation that the observed effects depend strongly on the CSR wake present in the drift and copropagating with the beam downstream of the bunch compression regions.

\section{EXPERIMENTAL METHOD}

The recirculation arcs, which are in a layout based on the Bates design [6], provide a simple method of adjusting the compression of the bunch. By changing the strength of four quadrupoles located in the first recirculation arc, the energy-dependent path length of the machine, the element $R_{56}$ in transport notation, may be adjusted. This allows for the compression point of a beam with a given energyposition correlation, or chirp, to be changed.

For these measurements $3.3 \mathrm{ps}$ long bunches at $9 \mathrm{MeV}$ and with a charge of $135 \mathrm{pC} /$ bunch were accelerated in the SRF linac sections up to $135 \mathrm{MeV}$. The acceleration was performed at phases $\pm 10^{\circ}$ from the maximum accelerating voltage. Other machine parameters relevant to this experiment are given in Table I. Measurements were conducted on both the rising side and falling side of the rf field. The falling side is peculiar in that it is an abnormal running condition for most FEL accelerators where the bunch compression chicane requires a positively chirped beam, at the chicane entrance, to achieve compression. ${ }^{1}$ A phase

\footnotetext{
${ }^{1}$ We use the convention that the head of the bunch lies at longitudinal position $s<0$ in the bunch-centered coordinate frame and that a standard chicane has $R_{56}<0$ making chirp $h>0$ necessary for compression.
}

$\pm 10^{\circ}$ provides a chirp such that $\frac{d E}{E d s}= \pm 5 \mathrm{~m}^{-1}$ and thus requiring $R_{56}=\mp 0.20 \mathrm{~m}$ to achieve full compression.

\section{A. Bunch compression for falling rf acceleration}

There are three areas which provide nonzero $R_{56}$; the first is the Bates arc (labeled A1 in Fig. 1) discussed previously, and the second is a standard four-dipole chicane (BC1 in Fig. 1) with $R_{56}^{\mathrm{BC} 1}=-0.52 \mathrm{~m}$. The last is the terahertz suppression chicane (BC2 in Fig. 1) [7], installed to alleviate the problem of heating of the downstream FEL optics due to terahertz CSR. The terahertz suppression chicane has $R_{56}^{\mathrm{BC} 2}=-4.6 \mathrm{~cm}$. It is of particular note because it appears to significantly impact our measurements only when care has been taken to remove any second-order curvature in the longitudinal phase space. This result is shown in a simulation later in the paper.

In the case of a negatively chirped bunch, in A1 the large negative compaction contribution from the second dipole dramatically increases the bunch length but maintains the sense of the chirp. As the bunch traverses the $180^{\circ}$ dipole, it is rapidly overcompressed, reversing the initial orientation of the head at high energy and the tail at low energy so that at the dipole exit the head is at low energy and the tail is at

TABLE I. Nominal beam parameters for the experiment and relevant characteristics of the machine.

\begin{tabular}{llc}
\hline \hline Symbol & \multicolumn{1}{c}{ Description } & Value \\
\hline$E_{0}$ & Injection energy [MeV] & 10 \\
$E_{f}$ & Final energy [MeV] & 135 \\
- & Charge per bunch [pC] & 135 \\
$\sigma_{0}$ & Bunch length after injector [ps] & 3.3 \\
$\sigma_{f}$ & Bunch length at max compression [fs] & 150 \\
$\mathrm{~h}$ & Energy-position correlation (chirp) $\left[\mathrm{m}^{-1}\right]$ & $\mp 5$ \\
- & rf phase [degrees] & \pm 10 \\
- & rf frequency [GHz] & 1.497 \\
$R_{56}^{\mathrm{BC} 1}$ & Optical cavity chicane $R_{56}[\mathrm{~cm}]$ & -52 \\
$R_{56}^{\mathrm{BC} 2}$ & Terahertz suppression chicane $R_{56}[\mathrm{~cm}]$ & -4.6 \\
$R_{56}^{\mathrm{A} 1}$ & Bates arcs $R_{56}[\mathrm{~cm}]$ & -7.5 to 85 \\
\hline \hline
\end{tabular}


high. After this rapid parasitic compression, the remainder of the transport to the second arc will then compress the bunch, with the location of the shortest bunch length depending on the $R_{56}^{\mathrm{A} 1}$ set point of the first arc.

If the compaction in A1 is large and positive (e.g., $\left.R_{56}^{\mathrm{A} 1} \sim 1 \mathrm{~m}\right)$, the downstream chicanes do not have adequately large negative compaction to fully compress the bunch; instead, it is fully compressed only when it reaches the second dipole of the second arc, where the large negative momentum compaction contribution from that dipole can serve to reduce the bunch length-in general, resulting in overcompression by the dipole exit. As in the first arc, the $180^{\circ}$ dipole then reverses the sign of the chirp, introducing yet another parasitic compression. If energy recovery is used, the compaction of the second arc must be adjusted to insure the overall recirculator transport line is isochronous so as to guarantee the bunch energy spread is compressed during energy recovery; this adjustment must be observant of the linac recovery phase and include the contribution of all dipoles along the beam line. We note that for the measurements discussed here, the machine was operated with tune beam and with a tune-up dump inserted at the end of the second arc — which was therefore not adjusted to track changes of the first arc and maintain isochronicity.

As the compaction of $\mathrm{A} 1$ is reduced from large positive values, the compression point moves upstream through the machine. At $R_{56}^{\mathrm{A} 1} \sim 0.75 \mathrm{~m}$, the bunch is fully compressed at the end of $\mathrm{BC} 2$; further reduction to $\sim 0.7 \mathrm{~m}$ moves the point to the end of $\mathrm{BC} 1$ just upstream of the wiggler. This is the normal configuration of the machine while lasing with positive linac-to-wiggler momentum compaction. We note that, for full compression at the end of the $\mathrm{BC} 1$, the bunch undergoes two parasitic compressions. The initial crossover is, as described above, in A1; the second crossover is in the third dipole of the $\mathrm{BC} 1$, where the bunch must be slightly overcompressed so as to ensure the bunch is fully upright after traversing the final dipole, which itself provides a small positive contribution to the overall transport compaction. Further reduction of the A1 compaction moves the compression point upstream. When $R_{56}^{\mathrm{A} 1}=0.2 \mathrm{~m}$, the bunch is fully compressed at the end of A1.

We note that this second parasitic crossover is unavoidable when using a chicane compressor and can be a cause for concern as the short bunch interacts with the forward propagating terahertz radiation field after exiting the third dipole. The crossover can be avoided through use of a compressor with monotonically increasing $R_{56}$, which can be made by using an arc beam line in which all dipoles bend in the same sense. In this case, the bunch is fully compressed only at the end of the final dipole; parasitic crossovers are entirely avoided.

\section{B. Bunch compression for rising $\mathrm{rf}$ acceleration}

A bunch with positive chirp will undergo a rapid parasitic compression as it traverses the large negative compaction contributed by the second dipole of A1. A second parasitic compression then soon follows as the bunch moves through the (large positive compaction of the) $180^{\circ}$ dipole and the head and tail are again reversed, restoring the initial positive chirp. The ensuing dynamics then - as above-depend on the compaction set point of A1. If $R_{56}^{\mathrm{A} 1}$ is large and positive, the bunch is, as above, fully recompressed only when it reaches the second dipole in the downstream Bates arc (A2 in Fig. 1). As the compaction is reduced, the compression point again moves forward, with the bunch being fully compressed between the $\mathrm{BC} 2$ and $\mathrm{A} 2$ when $R_{56}^{\mathrm{A} 1} \sim 0.35 \mathrm{~m}$. Further reduction to $R_{56}^{\mathrm{A} 1}=0.3 \mathrm{~m}$ puts the machine into the nominal lasing configuration, with the bunch fully compressed between $\mathrm{BC} 1$ and $\mathrm{BC} 2$ (and in the wiggler). As above, an additional parasitic compression, the third such, occurs in the third dipole of $\mathrm{BC} 1$ when the bunch is brought to full compression in this region.

Further reduction moves the compression point upstream through $\mathrm{BC} 1$, reaching full compression in the arc-tochicane nondispersed transport for $R_{56}=-0.2 \mathrm{~m}$. Additional reduction ensures that all compressions are then parasitic. It is of interest to note that, though the notional design dynamics of this system generate bunch compression by using a positive chirp, there is actually one fewer parasitic compression when the system is operated with acceleration on the falling side of the rf waveform. Preliminary beam operations in this configuration subsequently suggested that operation with a negative chirp may result in better system performance.

\section{Data collection}

As the quadrupoles in A1 were used to adjust the compression, beam position monitors (BPMs) on either side of the $180^{\circ}$ bend were used to record the average position of the beam. Measurement in this dispersive region

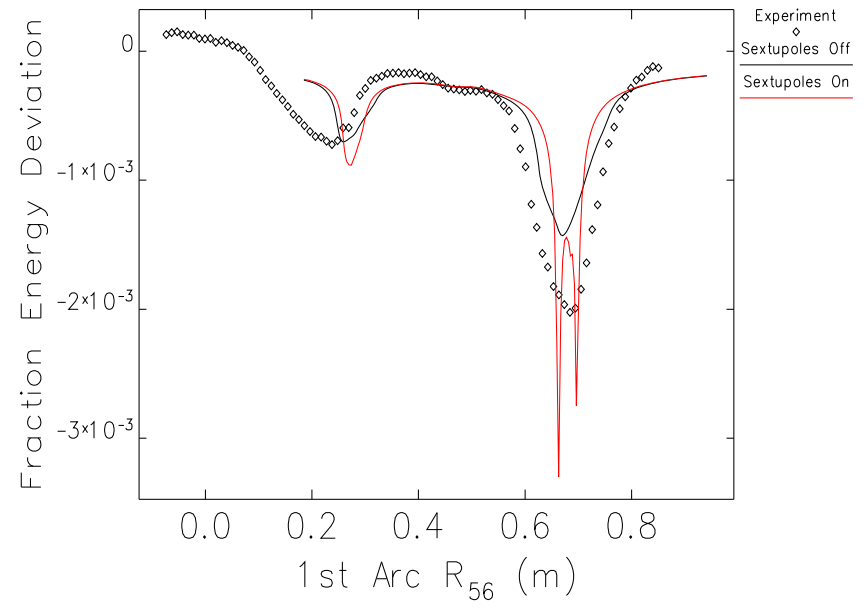

FIG. 2. Energy loss measurement and simulation due to CSR as a function of $R_{56}^{\mathrm{A} 1}$ for falling rf acceleration. 


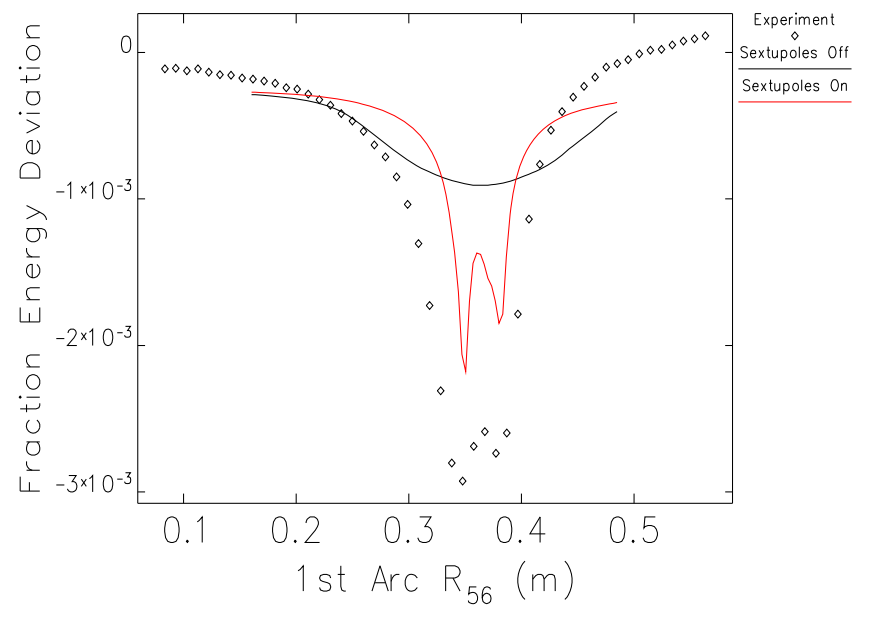

FIG. 3. Energy loss measurement and simulation due to CSR as a function of $R_{56}^{\mathrm{A} 1}$ for rising $\mathrm{rf}$ acceleration.

allows for direct observation of the average energy of the bunch. The $180^{\circ}$ bend also provides a system with point-to-point imaging and a magnification of -1 , which, by averaging the BPM readings on either side of the bend, will remove any betatron offset. This same measurement is performed in $\mathrm{A} 2$ on the opposite side of the machine where the beam position will have shifted based upon energy loss from CSR. By taking the difference between these two measurements, any common jitter in energy is removed, meaning any observed position change is only a function of the energy loss experienced between the two $180^{\circ}$ bend regions.

Shown in Fig. 2 is the average beam energy, as measured from the horizontal position of the BPMs in A2, as a function of $R_{56}^{\mathrm{A} 1}$ in $\mathrm{A} 1$. As expected, there are two compression points. The first occurs when the bunch fully compresses at the end of $\mathrm{A} 1$, and there is a large extraction of energy due to CSR within the final dipoles and in the following drift space. In this case, the bunch will overcompress within the chicane (BC1), and CSR losses are minimal there. The second is for the case when the bunch undergoes only parasitic compression in $\mathrm{A} 1$; this occurs rapidly and little energy is lost to CSR at this point. The bunch then fully compresses at the end of $\mathrm{BC} 1$ before the undulator. There is a large extraction of energy to CSR within the final two chicane dipoles when the bunch is fully compressed. The measurement taken with acceleration on the rising $\mathrm{rf}$ wave is shown in Fig. 3. During this measurement, the quadrupoles were not scanned over as broad a range, and only full compression within the chicanes is observed. A second dip due to full compression within A1 would be expected, though, had the scan range extended far enough.

\section{Energy spectra measurement}

A synchrotron light monitor placed in the $180^{\circ}$ dipole of A2 was used to take measurements of the bunch energy spectrum as the compression point was varied. These measurements were performed for just compression with falling side $\mathrm{rf}$ acceleration. During this process a distinctive "spike" forms in the energy spectrum and moves down along the spectrum; see Fig. 4. This occurs as the machine $R_{56}$ progresses from the point of undercompression at the end of $\mathrm{BC} 1$ to overcompression. This effect also is observed when the bunch is being compressed at the end of A1. This effect, in some ways, appears similar to the microbunching instability, which may occur in bunch compression chicanes; however, structures arising from microbunching in the bunch profile normally are shortwavelength structures and display a level of randomness as they arise from noise. The structure in the energy spectrum observed here is seen to move progressively from the high

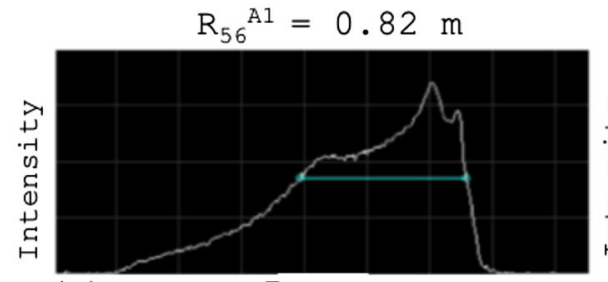

(a)

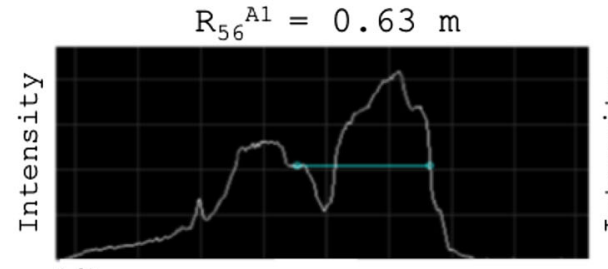

(d)

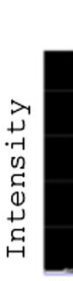

(b)

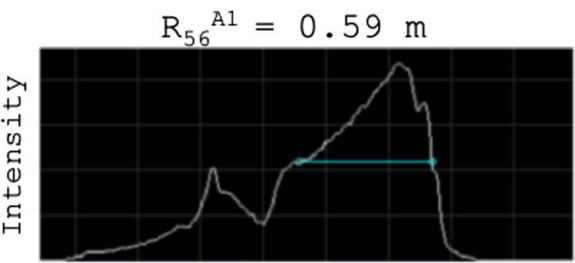

(e)

$\mathrm{R}_{56}^{\mathrm{Al}}=0.70 \mathrm{~m}$

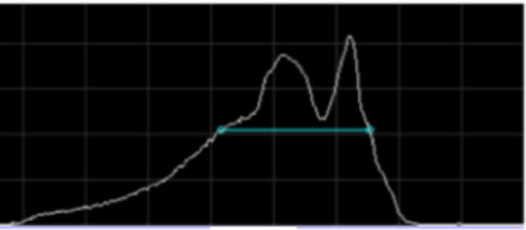

Energy

Energy

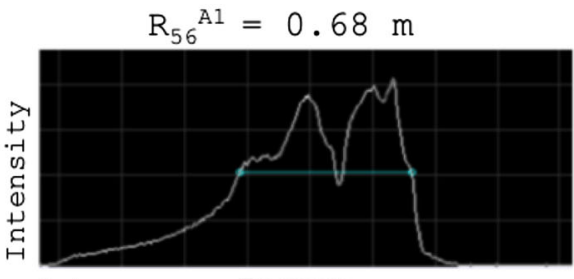

(c)

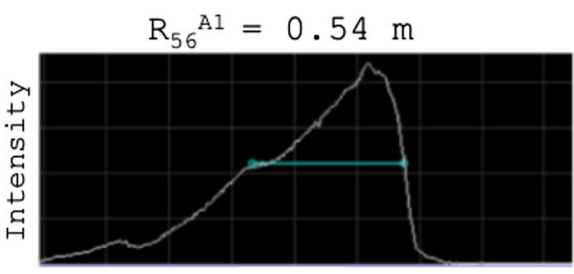

(f)

Energy

FIG. 4. Measurements of the energy spectrum collected in the second Bates arc. The vertical axis is the population (arbitrary units) and the horizontal axis is the energy (arbitrary units). As the compression is changed, a distinct spike and depletion region form and shift along the spectrum, shown from (a) to (f), as the result of CSR. 
energy to the low energy side of the spectrum in a predictable manner. Similar features have been observed in both simulation and measurement at various facilities, including during previous measurements on the JLAB FEL [8-10].

\section{SIMULATION}

Simulation of the accelerator starting after the injector was performed by using ELEGANT [11]. A 3.3 ps long bunch with a charge of $135 \mathrm{pC}$, modeled with 100000 macroparticles and a flattop distribution in the longitudinal plane and Gaussian distribution in both transverse planes is injected at $9 \mathrm{MeV}$ into the accelerating sections. Longitudinal space charge calculations were not included for these simulations. It should be noted that the bunch length was not measured during this experiment, but past measurements have given a value of around $3.3 \mathrm{ps}$. The simulation of the impact of the initial bunch length on CSR emission is shown in Fig. 5. It can be seen that, while there is the expected reduction in CSR from increasing the bunch length, there is little impact on the line shape of energy loss as a function of the machine compression point. Thus we will use the nominal length of 3.3 ps for all simulations shown.

The bunch is accelerated at $10^{\circ}$ off crest to give the proper time-energy correlation. As discussed previously, the quadrupole strengths in the first Bates arc (A1) are varied to adjust the compression point along the machine. The average energy loss accumulated up to the middle of the second Bates arc (A2) is shown in Figs. 2 and 3. Two simulation cases are considered; in one, the sextupoles within A1 were completely turned off. Good agreement with measurement is seen for the falling acceleration case (Fig. 2). The simulation was also run with the sextupoles set to remove beam curvature and provide a more linear

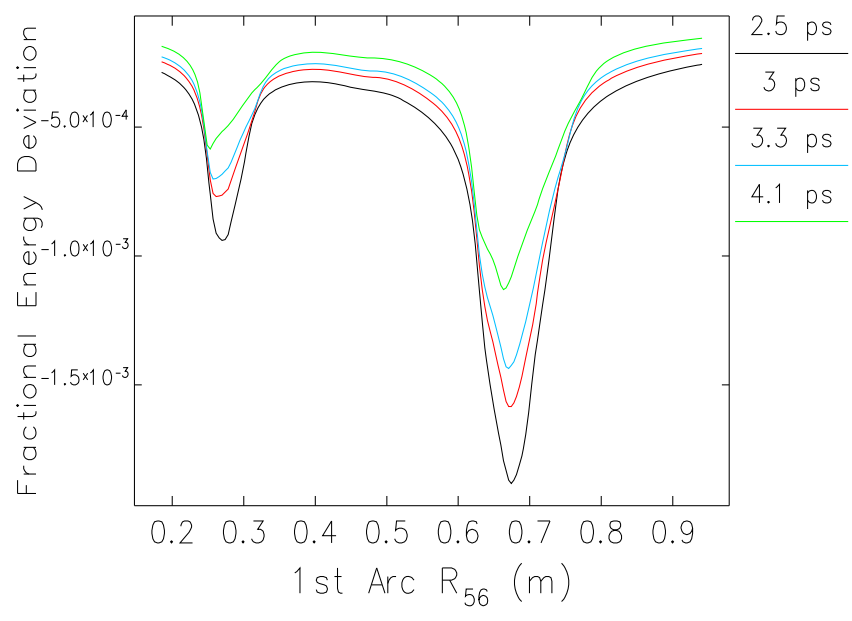

FIG. 5. Comparison of energy loss due to CSR in simulation for several different initial bunch lengths. The nominal bunch length value used in the simulations is 3.3 ps. compression at the end of the optical cavity chicane (BC1). The simulation of the falling rf acceleration case displays a pair of dips not seen in the experiment, but the pair of dips does appear in the rising rf measurement and simulation (Fig. 3). This suggests that the sextupoles were set poorly for the falling $\mathrm{rf}$ measurement but were tuned more properly for the measurement on the rising rf side. From the simulation it becomes clear that this twin dip structure arises from an undercompressed bunch entering the terahertz suppression chicane (BC2). The dip at higher $R_{56}$ corresponds to compression at the end of BC2. The dip at lower $R_{56}$, of the pair, occurs from compression at the end of BC1.

When the sextupoles have been properly set, the bunch is still very close to linearly chirped at the exit of $\mathrm{BC} 1$, allowing for the bunch to reach full compression in $\mathrm{BC} 2$; however, with the sextupoles off or set improperly, the bunch develops significant curvature after BC1 and experiences little further compression.

Shown in Fig. 6 is the energy spectrum of the beam observed at several points along the beam line and at various stages of compression. The same formation of a spike in the spectrum and movement with variation in the compression is evident. The simulation also demonstrates that the strength of this feature stems quite strongly from the CSR wake that is propagating with the beam after the chicane (Fig. 6). For simulation of CSR in the drift, the Stupakov model was used [12]. Because so much of the development of this spike takes place after the dispersive region of the bunch compressor, it should not be expected that this will lead to any longitudinal density modulation like that which occurs in the microbunching instability.

\section{A. Validity of the one-dimensional CSR model}

ELEGANT employs a 1D model of CSR [8] that assumes the horizontal extent of the bunch is much smaller than the bunch length. This requirement may be expressed [13] as $\kappa=\sigma_{\perp}\left(\frac{1}{R \sigma_{\|}^{2}}\right)^{\frac{1}{3}} \ll 1$, sometimes called the Derbenev criterion. Here $\sigma_{\|}$is the bunch length, $\sigma_{\perp}$ is the bunch size in the bending plane, and $R$ is the dipole bend radius. This corresponds to the assumption in the 1D model that propagation of the CSR wake is well modeled by projecting the longitudinal profile of the beam onto a line tangent to the bend; however, if there is a large transverse size, then particles that appear to be able to see the CSR wake from a certain point in the beam in the $1 \mathrm{D}$ model may in fact have too large a transverse separation for this to hold in an accurate three-dimensional description. Because of the comparatively large emittances for this machine, the Derbenev parameter can reach $\kappa>0.85$ for regions inside of dipoles where dispersion will be present and where the beam is being compressed. While this condition for validity has been violated, there still seems to be excellent qualitative agreement between the measurements and 1D CSR simulations. 

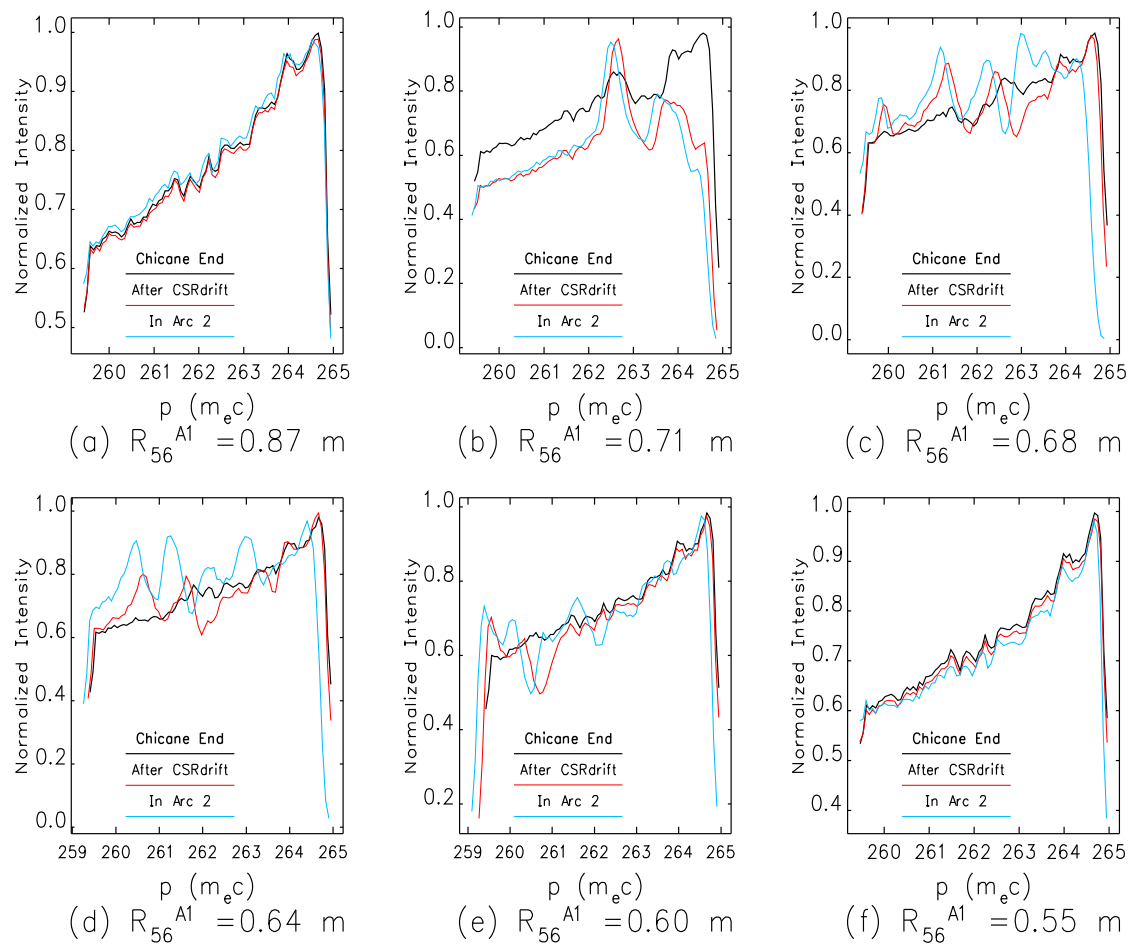

FIG. 6. Momentum spectra from simulation near full compression after the optical cavity chicane (BC1). The beam progresses from undercompression to overcompression in (a)-(f). The black line shows the spectrum immediately at the exit of the fourth dipole of the chicane, and the red line shows the spectrum after $4 \mathrm{~m}$ where CSR interaction with the bunch has continued. This interaction in the drift contributes significantly to the development of the spike and depletion regions.

\section{B. Impact of nonlinearity in the longitudinal phase space}

Important nonlinearities, relevant to bunch compression, and subsequently the intensity of CSR from the beam arise from a number of areas in the machine. As the bunch is accelerated, not only is the desired linear energy correlation established on the bunch, but a second-order correlation will also be imposed. This nonlinear correlation will be further amplified by the inherently nonlinear nature of the compression system of both the Bates arc and the compression chicane as is demonstrated in Fig. 7. This leads to undesirable effects with respect both to operation of a FEL and to minimizing CSR output from the beam. First, a curved bunch in longitudinal phase space will limit the minimum compressed bunch length and produce a strong leading spike of charge and a long tail that may still contain a significant portion of the charge. This formation is undesirable in regards to minimizing CSR, and as shown by Li [14] such structures may produce stronger CSR wakes when compared to a Gaussian distribution even when the rms bunch length is kept constant. To correct these effects, it is common to linearize the bunch and remove any higher-order longitudinal correlations. This is often accomplished by using a higher-order harmonic cavity [15]. The JLAB FEL, however, takes advantage of its two recirculation arcs to correct the higher-order moments of the beam during both compression and energy recovery. The use of sextupoles and octupoles for secondand third-order corrections, respectively, in the dispersive bending regions of the arcs removes nonlinearities first before compression and then in A2 to ensure proper conditions for energy recovery.

In our simulation the sextupoles of A1 were tuned to provide cancellation of the second-order curvature at the end of $\mathrm{BC} 1$. The condition for setting the sextupoles is derived from the condition that the bunch will first pass

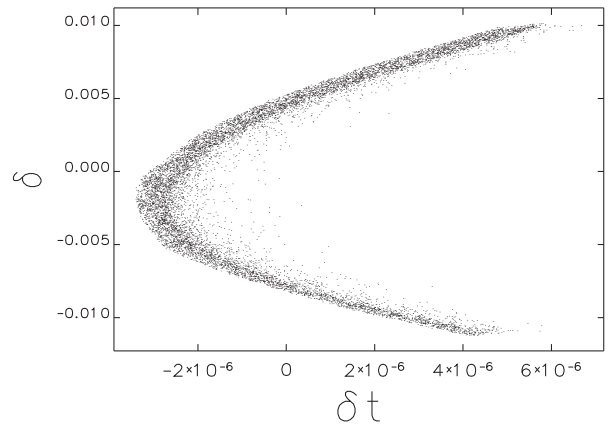

FIG. 7. Example of curvature in the longitudinal phase space induced on a compressed bunch from rf curvature and nonlinearity of the transport lattice. Here $\delta$ and $\delta t$ are normalized values of momentum and time, respectively. 
through the linac where the energy is modified as a function of position up to second order and then through the longitudinally dispersive region of the Bates arc and chicane. We may use the sextupoles in A1 to remove the second-order effect at the end of the chicane by optimizing their strength based on the following condition. In the cavity the longitudinal transfer functions are

$$
\begin{gathered}
z_{0} \rightarrow z_{1}=z_{0}, \\
\delta_{0} \rightarrow \delta_{1}=\delta_{0}+R_{65} z_{0}+T_{655} z_{0}^{2} .
\end{gathered}
$$

By placing this into the transfer function for the longitudinal position of a particle passing through a longitudinally dispersive region,

$$
\begin{aligned}
z_{1} \rightarrow z_{2}= & z_{0}+R_{56}\left(\delta_{0}+R_{65} z_{0}+T_{655} z_{0}^{2}\right) \\
& +T_{566}\left(\delta_{0}+R_{65} z_{0}+T_{655} z_{0}^{2}\right)^{2} .
\end{aligned}
$$

If we expand out this equation keeping only the secondorder term in $z$ whose coefficient we want to have equal to zero, we find

$$
R_{56} T_{655}+T_{566} R_{65}^{2}=0,
$$

where $R_{65}$ and $T_{655}$ are determined by the $\mathrm{rf}$ in the linac and $R_{56}$ is set primarily by the quadrupoles in $\mathrm{A} 1$ and by the design of BC1. The $T_{566}$ component may be controlled by adjusting the strength of the sextupoles in A1.

This linearization provides a more uniform current distribution and slightly higher peak current. Since the CSR wake is dependent on the current profile of the bunch, having a more uniform current distribution leads to CSR forces acting more uniformly across the bunch. The total energy loss to CSR more than doubles after BC1 (see Figs. 2 and 3) due to an increase in the peak current from when the bunch is properly linearized; however, with a correctly linearized bunch this will result in only a uniform shift of the energy distribution rather than the appearance of the originally observed nonuniformity.

To illustrate this, a series of simulations were run where the sextupole strength was increased incrementally up to $300 \%$ of the correct sextupole strength; for this procedure, the quadrupole strengths in A1 were held constant and set to provide maximum compression at the end of $\mathrm{BC} 1$. To measure the impact of the sextupoles on the energy spectrum, the spectrum at full sextupole strength was taken as a baseline and subtracted from each other energy spectra to normalize the spectra with the edges clipped. This is illustrated in Fig. 8(a), which shows the impact of the sextupoles on keeping the energy spectrum uniform. This deviation from a uniform distribution is quantified in Figs. 9(a) and 9(b), as the sextupole strength which shows the rapid growth of nonuniformities in the spectrum. Both the standard deviation and maximum deviation tend

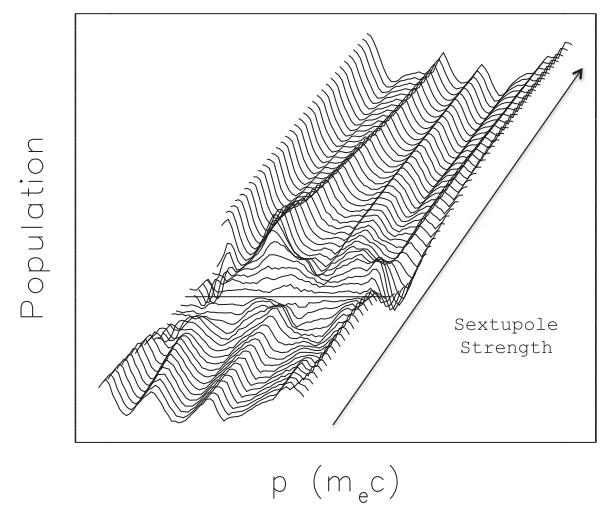

(a)

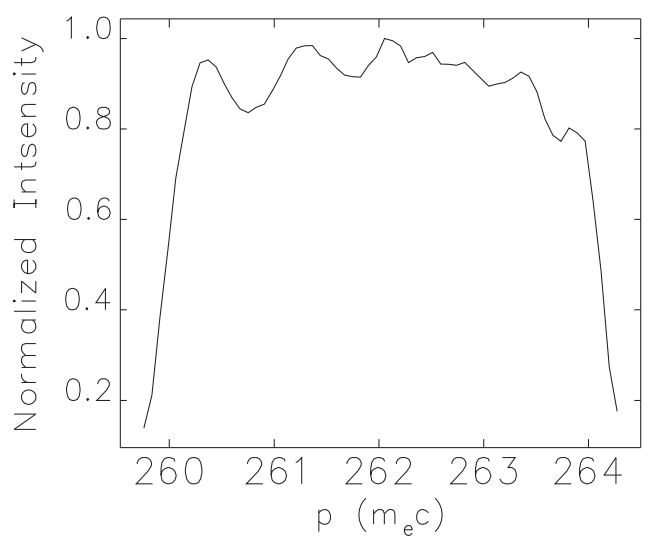

(b)

FIG. 8. (a) Mountain range plot of the energy spectrum difference from the baseline taken when the sextupoles are correctly set. The sextupole strength is scaled from 0 to $300 \%$ of the correct set point for linearizing the bunch. At the bottom left the sextupoles are completely off, while at the top right of the plot the sextupole strength has reach $300 \%$ of the correct set point. (b) Baseline momentum distribution when sextupoles are set to nominal strength.

to grow together, showing the formation of several large spikes. This is to be expected, as the CSR impact is localized in position and there still exists a second-order correlation between energy and position after $\mathrm{BC} 1$ when the sextupole correction is not used or is set improperly.

\section{Movement of the spike}

The formation of features does arise from the interaction of the strong CSR wakefield generated during compression which results in particles located in the region of the wakefield being shifted down in energy. This spatially localized shift is converted to a localized shift in the energy spectrum based on the correlation between particle position and energy which will be strongly nonlinear near full compression and in the absence of proper correction. This link between the spatial placement of the wake and the localization of its effect on a region of the energy spectrum 


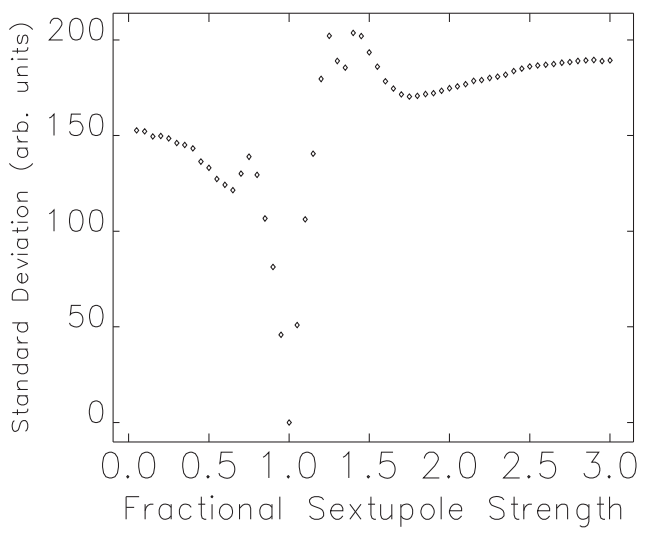

(a)

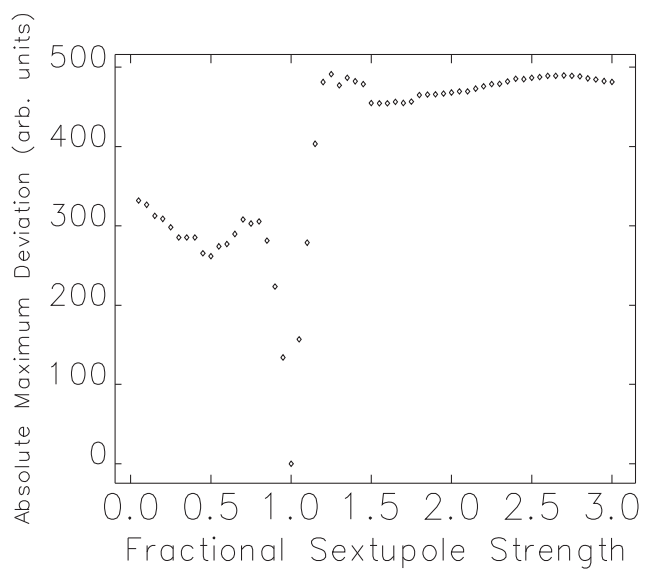

(b)

FIG. 9. The standard deviation (a) and the maximum deviation (b) of the energy spectrum minus the baseline as the sextupoles are scaled from 0 to $300 \%$ of the correct sextupole set point for linearizing a bunch at full compression after BC1. For both calculations, the edges of the distribution are excluded. can be seen just from the following simple analysis of the second-order longitudinal transformation of the bunch through a longitudinally dispersive region. The final longitudinal position $z_{1}$ with just longitudinal dispersive terms is

$$
z_{1}=z+R_{56} \delta+T_{566} \delta^{2}
$$

Assuming a linear chirp to start on the beam $h$, then $\delta=h z$, and neglecting radiative losses

$$
\delta=-\frac{\left(\frac{1}{h}+R_{56}\right)}{2 T_{566}} \pm \frac{1}{2 T_{566}} \sqrt{\left(\frac{1}{h}+R_{56}\right)^{2}+4 T_{566} z_{1}}
$$

The first term gives the location of the inflection point in $\delta$. Because of the nonlinear terms present from compression, which will be enhanced by any remaining curvature from the accelerating if field, the compression of the bunch will result in a spike in the current distribution at the head of the bunch. As the compression point is varied, the location of the head of the bunch will shift across the energy spectrum, and as a result the CSR wake generated by the spike in the charge distribution will most strongly affect a small region of the bunch's spectrum. Such a non-Gaussian spike can result in a much stronger CSR wake than from a Gaussian-shaped charge distribution and will produce the observed spike and depletion regions observed in the energy spectrum. This calculation is shown in Fig. 10 by the horizontal line and shows reasonable agreement with the simulation in predicting the region affected by the CSR wake.

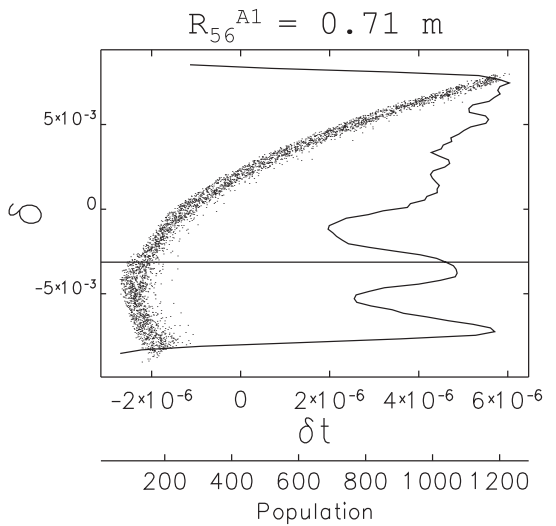

(a)

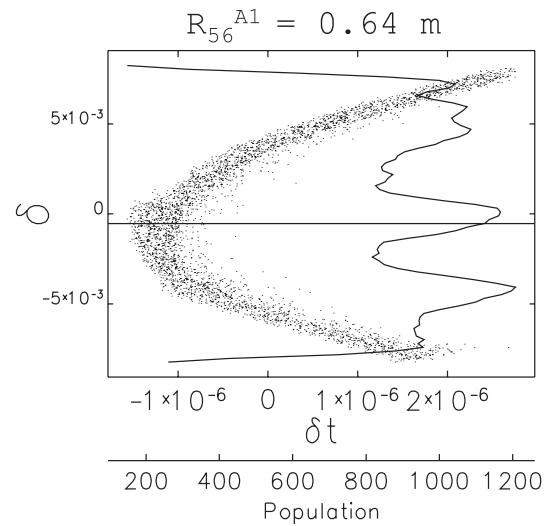

(b)

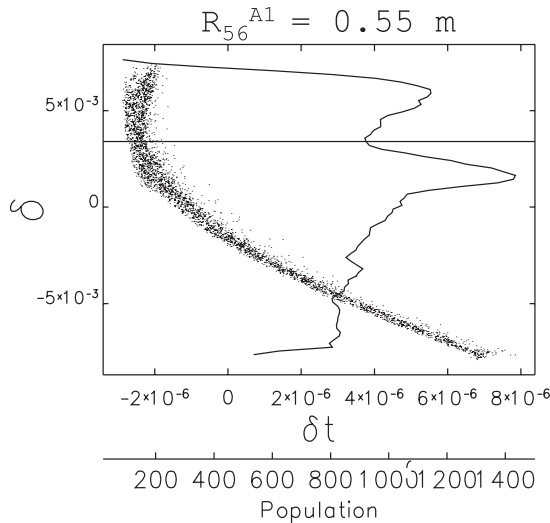

(c)

FIG. 10. Plots from simulation of the longitudinal phase space overlayed with the momentum spectrum. Where $\delta$ and $\delta t$ are normalized values of momentum and time respectively. From left to right shows the progression of: (a) undercompression, (b) full compression, and (c) overcompression within $\mathrm{BC}$. The horizontal line running across the plot shows the calculated location where the spike and depletion in each spectrum should occur. 


\section{CONCLUSION}

We have shown the results of measurements of the impact of CSR on the electron beam quality of the JLAB IR FEL as it varies with bunch compression. Very good agreement is seen between these measurements and simulations performed by using a 1D CSR model (in ELEGANT). This provides the observation of the bunch energy spectrum with varying compression and shows the systematic formation of spikes that shift in a regular fashion across the span of the spectrum as compression is varied. Based on simulations of the machine, it appears that this formation is strongly enhanced by CSR radiation propagating with the beam downstream of the final dipole of the compression region. The movement of the spike as compression is varied may be explained by looking at the quadratic transport equation for the longitudinal phase space in a longitudinally dispersive region.

From the simulation we can also see that control of the longitudinal curvature of the bunch is essential for properly controlling the behavior of the beam in regards to compression, maintaining the energy spectrum of the bunch, and preventing modulation. It would be desirable to make additional measurements further examining the impact of CSR on the beam. In particular, verifying the impact of the sextupoles (as shown in simulation) on reducing the modulation of the energy spectrum by CSR would be a very interesting study. Unfortunately, the JLAB FEL has been shut down due to funding issues since shortly after these measurements were taken in 2012, and we do not anticipate being able to continue this work in the foreseeable future.

\section{ACKNOWLEDGMENTS}

We thank J. Lewellen for proofreading of this manuscript and for his insightful comments on this work. We also thank P. Evtushenko for his invaluable assistance during the machine measurements. This work was funded by the Office of Naval Research and the High-Energy Laser Joint Technology Office.

[1] D. Douglas, S. V. Benson, G. A. Krafft, R. Li, L. Merminga, and B. C. Yunn, Driver Accelerator Design for the $10 \mathrm{~kW}$ Upgrade of the Jefferson Lab IR FEL, in Proceedings of LINAC2000 (SLAC, Monterey, CA, 2000), pp. 857-859.

[2] S. Benson et al., High power operation of the JLAB IR FEL driver accelerator, in Proceedings of the 22nd Particle Accelerator Conference, PAC-2007, Albuquerque, NM (IEEE, New York, 2007), pp. 79-81.
[3] E. Saldin, E. Schneidmiller, and M. Yurkov, On the coherent radiation of an electron bunch moving in an arc of a circle, Nucl. Instrum. Methods Phys. Res., Sect. A 398, 373 (1997).

[4] M. Borland, Y. C. Chae, S. Milton, R. Soliday, V. Bharadwaj, P. Emma, P. Krejcik, C. Limborg, H. Nuhn, and M. Woodley, Start-to-end jitter simulations of the linac coherent light source, in Proceedings of the 19th Particle Accelerator Conference, Chicago, IL, 2001 (IEEE, Piscataway, NJ, 2001), p. 2707.

[5] S. Heifets and G. Stupakov, Beam instability and microbunching due to CSR, in Proceedings of the 19th Particle Accelerator Conference, Chicago, IL, 2001 (IEEE, Piscataway, NJ, 2001).

[6] J. B. Flanz, and C. P. Sargent, Operation of an isochronous beam recirculation system, Nucl. Instrum. Methods Phys. Res., Sect. A 241, 325 (1985).

[7] D. Douglas, A THz suppression chicane for the FEL upgrade energy recovery transport (IR upgrade design revision 113b), JLAB Technical Report No. JLAB-TN04-028, 2004.

[8] M. Borland, Simple method for particle tracking with coherent synchrotron radiation, Phys. Rev. ST Accel. Beams 4, 070701 (2001).

[9] H. Braun, R. Corsini, L. Groening, F. Zhou, A. Kabel, T. O. Raubenheimer, R. Li, and T. Limberg, Emittance growth and energy loss due to coherent synchrotron radiation in the bunch compressor of the CLIC test facility (CTF II), in Proceedings of the European Particle Accelerator Conference, Vienna, 2000 (EPS, Geneva, 2000), pp. 471-473.

[10] P. Piot, C. L. Bohn, D. R. Douglas, G. A. Krafft, R. Li, and B. C. Yunn, Emittance and energy spread studies in the Jefferson Lab free-electron laser, in Proceedings of the European Particle Accelerator Conference, Vienna, 2000 (EPS, Geneva, 2000), pp. 1546-1548.

[11] M. Borland, elegant: A Flexible SDDS-Compliant Code for Accelerator Simulation, Report No. LS-287, ANL, 2000.

[12] G. Stupakov and P. Emma, CSR wake for a short magnet in ultrarelativistic limit, SLAC Technical Report No. LCLSTN-01-12, 2001.

[13] Y.S. Derbenev, J. Rossbach, E. L. Saldin, and V.D. Shiltsev, Microbunch radiative tail-head interaction, TESLA Technical Report No. TESLA-FEL 95-05, 1995.

[14] R. Li, Sensitivity of the CSR self-interaction to the local longitudinal charge concentration of an electron bunch, Nucl. Instrum. Methods Phys. Res., Sect. A 475, 498 (2001).

[15] D. Dowell et al., Magnetic pulse compression using a third harmonic linearizer, in Proceedings of the Particle Accelerator Conference, Dallas, TX, 1995 (IEEE, New York, 1995). 